\title{
Enablers for Energy Efficient Wireless Networks
}

\author{
(Invited Paper) \\ Gunther Auer*, István Gódor ${ }^{\dagger}$, László Hévizi ${ }^{\dagger}$, Muhammad Ali Imran ${ }^{\ddagger}$, Jens Malmodin ${ }^{\S}$, \\ Péter Fazekas ${ }^{\rrbracket}$, Gergely Biczók ${ }^{\rrbracket}$, Hauke Holtkamp*, Dietrich Zellerl, Oliver Blumell, Rahim Tafazolli ${ }^{\ddagger}$ \\ *DOCOMO Euro-Labs, 80687 Munich, Germany, Email: lastname@docomolab-euro.com \\ ${ }^{\dagger}$ Ericsson Research, Budapest $\mathrm{H}-1117$, Hungary \\ ${ }_{\ddagger}$ CCSR University of Surrey, Guildford GU2 7XH, UK \\ $\S$ Ericsson Radio Systems, Stockholm, Sweden \\ I Budapest University of Technology and Economics, Budapest H-1117, Hungary \\ "Alcatel-Lucent, Bell Labs Germany, 70435 Stuttgart, Germany
}

\begin{abstract}
Mobile communications are increasingly contributing to global energy consumption. The EARTH (Energy Aware Radio and neTworking tecHnologies) project tackles the important issue of reducing $\mathrm{CO}_{2}$ emissions by enhancing the energy efficiency of cellular mobile networks. EARTH is a holistic approach to develop a new generation of energy efficient products, components, deployment strategies and energy-aware network management solutions. In this paper the holistic EARTH approach to energy efficient mobile communication systems is introduced. Performance metrics are studied to assess the theoretical bounds of energy efficiency as well as the practical achievable limits. A vast potential for energy savings lies in the operation of radio base stations. In particular, base stations consume a considerable amount of the available power budget even when operating at low load. Energy efficient radio resource management (RRM) strategies need to take into account slowly changing daily load patterns, as well as highly dynamic traffic fluctuations. Moreover, various deployment strategies are examined focusing on their potential to reduce energy consumption, whilst providing uncompromised coverage and user experience. This includes heterogeneous networks with a sophisticated mix of different cell sizes, which may be further enhanced by energy efficient relaying and base station cooperation technologies. Finally, scenarios leveraging the capability of advanced terminals to operate on multiple radio access technologies (RAT) are discussed with respect to their energy savings potential.
\end{abstract}

\section{INTRODUCTION}

The global mobile communications industry is growing rapidly. Today there are more than four billion mobile phone subscribers worldwide, more than half the entire population of the planet. Obviously, this growth is accompanied by increased energy consumption of mobile networks. The objective of the EARTH project is to address the global environmental challenge by identifying effective mechanisms to substantially reduce energy wastage and to improve energy efficiency of mobile communication systems, without compromising network coverage and users perceived quality of service.

In order to quantify the merits of the envisaged energy savings, a holistic system view will be adopted, ensuring that any radio energy efficiency improvement in one area is not wiped out by an increased energy consumption in a different area, e.g. gains at the base station are not neutralized in the core network or at the user terminal. While the performance of radio access technologies is typically characterized by metrics like spectral efficiency, throughput or coverage, these metrics are unable to quantify the energy efficiency of a network. A thorough analysis of key radio and network technologies in terms of their energy efficiency is not yet available. This is partly due to the fact that there are no suitable methodologies and no sophisticated metrics available that enable a fair and objective evaluation of the entire system, ranging from high power efficiency at the base station site - including the necessary air conditioning — to alternative deployment strategies, such as relaying concepts, heterogeneous networks and base station cooperation. Thus, an important prerequisite for efforts to enhance the energy efficiency of cellular networks is to provide a framework to quantify the attainable energy savings.

Today's mobile networks have a vast potential for energy savings. While the energy efficiency of mobile terminals is highly optimized due to the stringent constraints on the available power supply, until recently power consumption of base stations has been largely ignored. This has led to a situation where terminal energy consumption is only a fraction of the energy consumption of the mobile network, while base stations are the major source of $\mathrm{CO}_{2}$ emissions [1,2]. Consequently, the EARTH project has put its focus on enhancing the energy efficiency of radio base stations. In particular, even at low load base stations consume a considerable amount of the available power budget.

Cellular networks exhibit slowly changing daily load patterns as well as highly dynamic traffic fluctuations. However, current networks are configured rather statically. Since most of the energy wastage occurs during low load situations, these load variations can be effectively exploited to reduce network energy consumption. Vendors and operators have started to exploit these load variations by bundling traffic in certain transceivers and switching others off. However, such algorithms are at an early stage and with the emergence of self optimizing networks (SON), there is an emerging potential to react on slowly changing traffic variations. Similarly, highly dynamic load fluctuations may be addressed by energy efficient radio resource management (RRM) strategies. EARTH 
will contribute with concepts and algorithms to significantly reduce the energy consumption in low load situations.

One further source of energy wastage is the layout of today's cellular networks. Obviously, a network layout optimized for coverage does not necessarily maximize energy efficiency. EARTH aims at devising fundamental principles of energy efficient network layouts. In particular we will investigate the use of technologies beyond the state of the art, such as advanced repeaters, relaying solutions and heterogeneous deployments that deviate from the conventional cellular deployment paradigm. This includes heterogeneous networks with a sophisticated mix of different cell sizes, optimized for minimal energy consumption. Ultimately, this leads to novel energy efficient network architectures.

In this paper the challenges and key enablers for energy efficient wireless networks are described. A discussion of the key levers for energy efficient radio components is provided in a companion paper [3]. The impact of mobile communication systems on the global $\mathrm{CO}_{2}$ emission is discussed in Section II. Moreover, the major sources of energy wastage are identified. Section III discusses appropriate energy efficiency metrics that enable a holistic system level comparison of competing technologies. SectionIV and V outline the potential of RRM strategies and alternative network deployments that aim to complement the cellular network layout paradigm. Section VI elaborates on energy savings envisaged by terminals that are able to operate on multiple radio access technologies.

\section{Socio-Economic Impact and Life Cycle Analysis}

As of today, $\mathrm{CO}_{2}$ equivalents $\left(\mathrm{CO}_{2}\right.$-eq) are generally used to measure the potential global warming contributions from organizations and products, e.g. in environmental research, societal push (e.g. Kyoto protocol), and in the media. The largest source of $\mathrm{CO}_{2}$-eq emissions are fossil fuels. It has become the most common and understandable metric, allowing to directly compare the contribution (the carbon footprint) of a given technology sector or organization with other contributions and reduction targets. When calculating carbon footprints, a holistic approach such as life cycle analysis (LCA) is recommended, rather then simple methods that only look at the use phase.

\section{A. Global carbon footprint of wireless communications}

Today, Information and Communication Technology (ICT) systems including data networks, fixed and wireless networks, as well as all end-user equipment such as PCs, home network equipment and mobile devices, are responsible for about $2 \%$ of global $\mathrm{CO}_{2}$ emissions and about $1.5 \%$ of global $\mathrm{CO}_{2}$-eq emissions, as shown in Fig. 1. In order to tackle climate change the EU Commission recently issued a call on ICT industry for intensified efforts to reduce its carbon footprint by $20 \%$ as early as 2015 for [4]. The mobile part of ICT is responsible for about $0.2 \%$ of global $\mathrm{CO}_{2}$-eq emissions but the number of radio base station (RBS) sites and mobile subscribers are steadily increasing (4 billion subscribers right now) and data-intensive applications are proliferating. Obviously, this

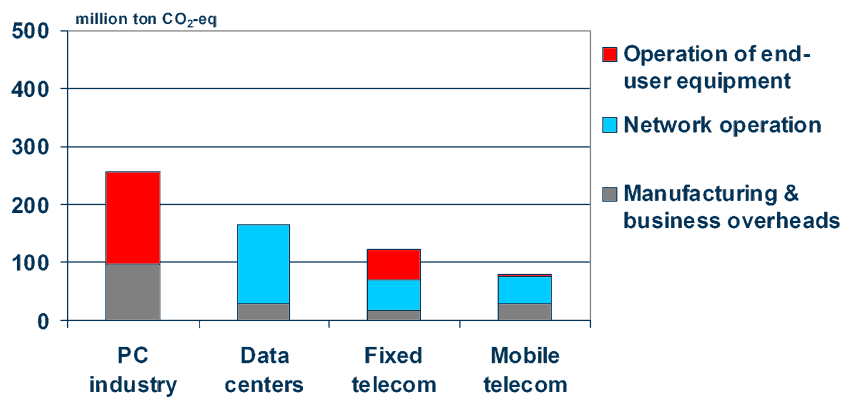

Fig. 1. $\mathrm{CO}_{2}$-eq emissions for the different ICT subsectors in 2007. Data centers also include data networks and data transport.

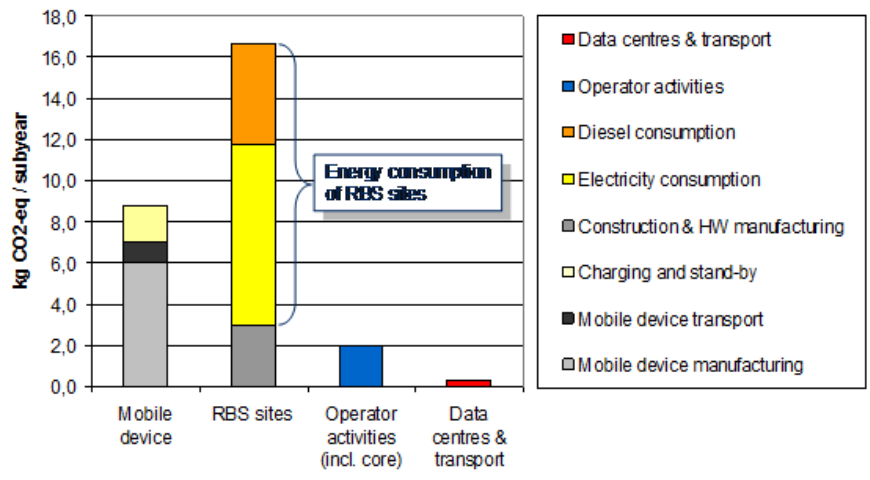

Fig. 2. The carbon footprint $\left(\mathrm{CO}_{2}\right.$-eq emissions) for an average mobile subscriber in 2007.

growth is accompanied by an increased energy consumption of mobile networks, with a corresponding increase in the carbon footprint [5].

On the other hand, rising energy costs, especially for networks where a large share of the RBS sites runs on diesel generators, have led to a situation where such sites contribute significantly to the network operation costs. In fact, operator's OPEX figures indicate that their energy costs are nowadays comparable to their personnel costs for network operations.

\section{B. Life cycle analysis}

Fig. 2 shows the carbon footprint for an average mobile subscriber, based on data for the full year 2007. The carbon footprint is based on extensive LCA studies covering the whole life cycle of the various components that are part of a mobile network.

It can be clearly seen that the energy consumption of RBS sites is responsible for the largest part of the total life cycle carbon footprint, followed by the mobile phone manufacturing. As a majority of sites has low traffic most of the time, this "stand-by" behavior of the network has a large potential for energy savings in the future.

An important part in an LCA of mobile networks is to assess the emissions from the electricity production sources. A world average electricity production model is used in the ICT and mobile network study presented here. The resulting emission 


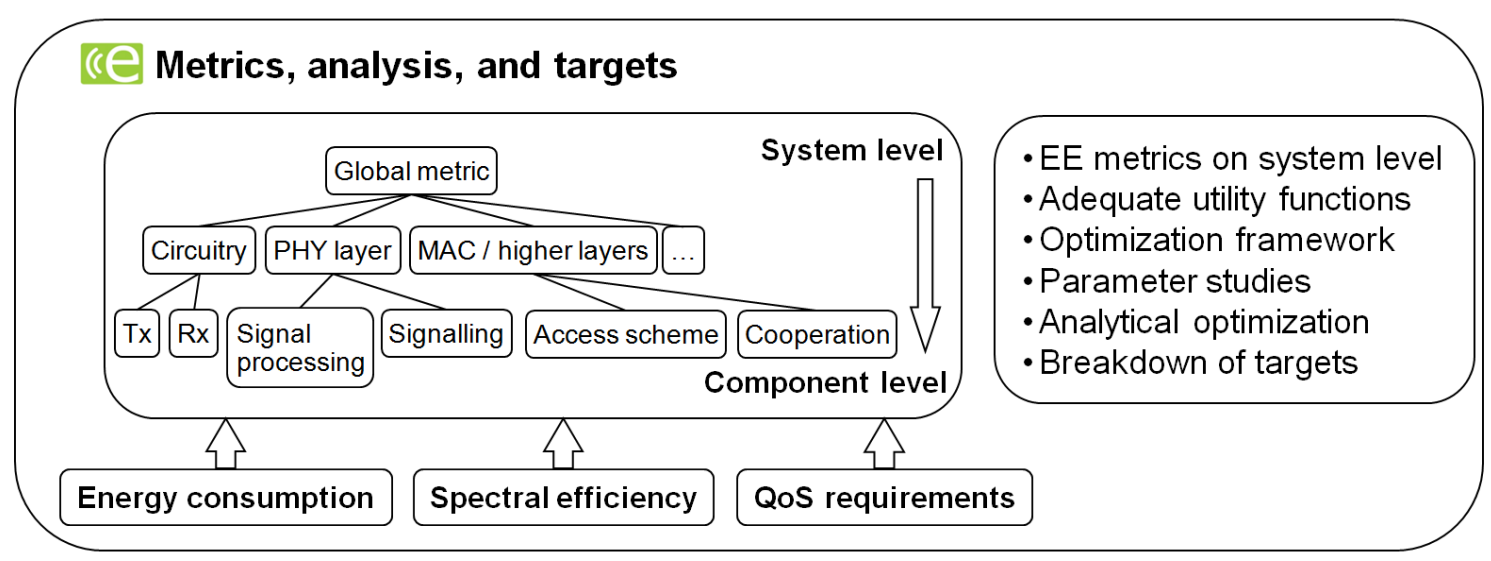

Fig. 3. EARTH approach towards a global energy efficiency metric.

factor is $0.6 \mathrm{~kg} \mathrm{CO}_{2}$-eq $/ \mathrm{kWh}$, where $0.5 \mathrm{~kg} \mathrm{CO} \mathrm{CO}_{2}$ comes from the direct production at the power plants.

The carbon footprint for an average mobile subscriber has decreased over time from more than $100 \mathrm{~kg} \mathrm{CO}_{2}$ per subscriber per year in the early 90's to approx. $25 \mathrm{~kg}$ in the mid 00 's. However, the increased number of RBS sites operated on diesel, "growing" mobile devices, and the rapid growth of new mobile networks has reversed this positive trend.

How much $\mathrm{CO}_{2}$ is $25 \mathrm{~kg}$ ? It equals to the amount emitted when 10.5 liter petrol is incinerated in a petrol engine, which takes you about 1 hour or $120 \mathrm{~km}$ on the motorway.

\section{ENERGY EFFICIENCY METRICS}

The classical optimization criterion for wireless network is the areal spectral efficiency, measured in $\left[\mathrm{bit} / \mathrm{s} / \mathrm{Hz} / \mathrm{m}^{2}\right]$. This implies maximizing the data rate per unit area for a given transmit power budget. Conventional energy efficiency metrics typically capture only a small fraction of the overall power budget of wireless networks, and may therefore lead to incomplete and potentially misleading conclusions. To this end, an appropriate energy efficiency metric should take into account the following components:

- The input power required to generate a specific output power at the antennas. This input power accounts for the desired power in analogue front-end, the power amplifier losses, air conditioning power consumption within base stations and the power loss of the feeder cables.

- The energy that is consumed by running the digital signal processing equipment (e.g. powerful channel coding with the associated complex decoders).

- The impact of the RRM scheme on the energy efficiency; such as subcarrier and transmit power allocation and base station cooperation (energy required for the exchange of message signals as well as additional control signaling).

- The energy to deliver data to the base station (backhaul power consumption).

A system level view requires a global energy efficiency metric, which is composed of specific metrics on the component level, as illustrated in Fig. 3. The challenge in devising a suitable energy efficiency metric lies not only in the dimension of the problem space, but also in the fact that the power consumption of the relevant components is interdependent.

Standardization of energy efficiency metrics has already commenced. For instance the ETSI Technical Committee on Environmental Engineering (ETSI-TC-EE) [6] has initiated work on a specification of energy efficiency metrics for mobile cellular networks. However, these specifications only consider energy efficiency metrics on component level (e.g. power efficiency for high power amplifiers) rather than the whole system (end-to-end connectivity including backhaul). Furthermore, criteria related to the quality-of-service are neglected.

For measuring the efficiency of the communication link, several metrics are used in the literature. The most commonly used metric for the energy efficiency of a communication link is bit per joule [7], which is an information theoretic measure of the energy efficiency of delay insensitive communication. To account for the data rate as well the communication distance, a modified metric of bit meter per joule may be used. This metric describes the efficiency of reliably transporting the bits over a distance towards the destination per unit of energy consumed [8]. For a cellular area of coverage, this metric is to be modified to bits per joule per unit area, so to capture the extent of the coverage area.

The system level energy efficiency of a single cell was studied in [9], where the energy efficiency of communicating over a frequency selective cellular uplink channel was optimized. The authors conclude that both the circuit energy (energy required to keep nodes active) and transmission energy need to be modeled, so to measure the overall energy efficiency in terms of bit per joule.

There exist a multitude of individual figures of merit for optimizing the different aspects and components of wireless systems (e.g. spectral efficiency, power consumption for data transmission and processing, quality of service parameters such as throughput, error rate, delay (jitter), coverage). It is indispensable that an energy efficiency metric takes all of the aforementioned aspects into account; otherwise the optimization might just improve towards one direction, at 
the expense of other important parameters. In this regard the EARTH project is developing more sophisticated energy efficiency metrics in the form of desired utility per unit of energy consumption covering a unit area of cellular coverage. The desired utility is to be calculated using a utility function capturing an appropriate mix of the above mentioned figures of merit.

\section{Radio Resource Management}

An essential component of energy efficient operation is that the network management adapts the network configuration and topology to the daily, spatial variation of traffic demand. In GSM/WCDMA networks, the state-of-the-art is to turn on and off some selected transceivers, cells and the associated backhaul links, e.g., during low-traffic night hours [10]. The EARTH project will provide new network management concepts for dynamic reconfiguration of radio networks (e.g. self-optimization, self-configuration and standby-operation). This will allow operators to utilize the different network deployment concepts (e.g. optimal mix of cell sizes, multiRAT deployments) and to dynamically exploit the potentials in reduction of energy consumption.

In order to save energy, the management procedures should concentrate on the fact that during low traffic hours it is the coverage requirement, in peak hours it is the capacity requirement, which determines the topology. As a consequence of the slow daily variation of capacity requirements, the (self) adaptation is assumed to operate on a longer time scale (minutes, hours) compared to radio resource management (RRM) (below seconds).

It is also important to identify situations where the reconfiguration of a system component or the coordinated reconfiguration of a group of components may save energy. Such situations arise when the antenna tilt is adapted to modify cell coverage, or by switching between various MIMO functionalities, etc. In addition, the network should be enabled to point the capacity into the place of need, e.g. by directing adaptive antenna arrays towards a traffic hotspot.

Cooperation mechanisms between base stations will be studied from an energy efficiency perspective. The transmission power of individual base stations may be reduced due to increased coverage, increased spectral efficiency, and adaptation to load variations between cells. On the other hand, energy consumption may increase due to more complex signal processing and/or additional backhaul traffic. Fundamental trade-offs between these factors will be studied providing the basis for designing energy efficient site cooperation protocols. As cooperating base stations resemble a distributed MIMO system, studies for energy saving potentials of MIMO systems provide a good starting point [11]. User cooperation for energy efficiency is studied in a simplistic two user scenario in [12].

\section{EnERgy EFFICIENT Network DePloyment}

Both environmental concerns and operational cost efficiency recently diverted the attention of wireless broadband research activities towards energy efficient deployment. Recent studies

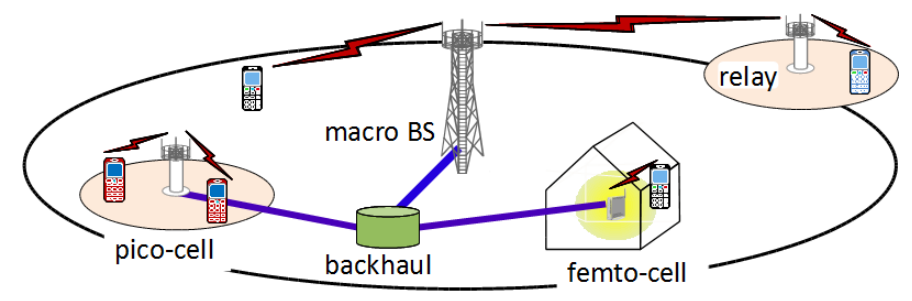

Fig. 4. Heterogeneous network deployment composed of macro-cells that provide wide area coverage, micro and pico-cells serving hot spots, femtocells that are deployed indoors, and relays.

define specific scenarios that have potential to improve the system energy efficiency. An overview of some promising approaches for improved energy efficient wireless access is provided in $[1,2,13,14]$.

In addition to energy efficient base station and core network design, emerging deployment strategies are to be considered that incorporate heterogeneous networks with cells of various size, ranging from macro-cells, over micro and pico-cells serving hot spots, to femto-cells that are deployed indoors, as shown in Fig. 4. The short distance between transmitter and receiver offered by small cell sizes suggest an enormous potential for energy savings; in turn, however, at low traffic loads the increased number of base stations at standby mode add to the overall power consumption, which may cancel parts of these envisaged energy savings. Hence an optimal balance must be found leading to the most energy efficient deployment scenario. In the EARTH project, the energy consumption to maintain network operation will be evaluated as a function of cell size and deployment scenario for given system loads and environments, ranging from dense urban to rural areas. Several deployment options like sectorization, antenna tilting and distributed antennas also need to be considered. Some initial work in this area is reported in [15-18].

Heterogeneous networks are complemented by relays, repeaters and cooperating base stations for the most energy efficient mix of different deployment strategies. Relay and repeater nodes are often promoted for infrastructure cost saving reasons, since expensive backhaul links are avoided. Another potential advantage is energy saving, since the propagation distance per hop (and thereby transmission power) is decreased [19]. These and other potential energy efficiency saving features [20] of relays and repeaters will be further explored within the EARTH project.

\section{Management of Multi-RAT Networks}

Currently base stations of multiple radio access technologies (RAT) are usually colocated and several RATs may cover the same area, but they provide different levels of services (cf. GSM, 3G, WLAN). With the projected roll-out of the 3GPP long term evolution (LTE) network, the number of available radio interface technologies further increases.

Multi-RAT terminals may take advantage of coexistence of different RATs. Unfortunately, the present practice is that each RAT has its own network planning, operations \& maintenance 
and resource management methodologies. Recent efforts from operators and vendors, e.g. carried out within the EU project Ambient Networks [21], aim at joint management of multiRAT technologies, focusing on capacity and coverage optimization.

In order to harvest energy savings from multi-RAT technologies new network functions are needed, to facilitate the dynamic distribution of load among interfaces (without any user intervention), to support the parallel use of multiple interfaces for the same data transfer (e.g. large file download), and to enable smooth switching of customer traffic between interfaces. Specifically, radio resource management (RRM) is to be enabled to utilize multiple air interfaces as a common resource. EARTH will develop deployment and operating strategies on how the network of different RATs can be deployed and operated in a coordinated fashion.

In order to reduce the energy consumption in multi-RAT deployments, as rule of thumb, the RAT with the lowest carrier frequency should be selected to maintain coverage at minimal power dissipation, due to the favorable propagation conditions at lower frequencies. Along this line, very low power and low coverage RATs (e.g. LTE femto cells) that typically operate in higher frequency bands should cover higher traffic demand areas. In this latter case, the short distance between transmitter and receiver overcompensates the energy efficiency loss of the high carrier frequency.

Naturally, the goals of EARTH go beyond the above examples and try to find the best network management solutions, which provide a joint optimization of multi-RAT networks.

\section{CONCLUSIONS}

Beyond today's focus of network element design, the European research project EARTH develops a holistic approach for the design of energy efficient mobile communication networks of the future. Energy efficiency metrics, deployment strategies and radio resource management are identified as key research areas beyond the radio node level research [3].

The project will address both the operational impacts energy efficient mobile radio and the impacts on climate change. We believe that cost saving can make ICT services sustainable both energetically and economically, thus, allowing further reduction of the global $\mathrm{CO}_{2}$ footprint, e.g. by teleconferencing, telecommuting and dematerialization. The ambitious goal of EARTH is to cut the energy consumption of mobile broadband networks by at least $50 \%$.

The results of EARTH will be introduced to standardization bodies and industry product development in order to ensure impact of the project to the technical community.

\section{ACKNOWLEDGEMENTS}

The authors gratefully acknowledge the contribution of the EARTH consortium to the technical definition of the project.
The work leading to this paper has received funding from the European Community's Seventh Framework Programme [FP7/2007-2013] under grant agreement $n^{\circ} 247733$ — project EARTH.

\section{REFERENCES}

[1] M. Gruber, O. Blume, D. Ferling, D. Zeller, M. A. Imran, and E. Calvanese-Strinati, "EARTH - Energy Aware Radio and Network Technologies," in Proc. IEEE International Symposium on Personal, Indoor and Mobile Radio Communications (PIMRC), Cannes, France, 2008.

[2] J. T. Louhi and H.-O. Scheck, "Energy efficiency of cellular networks," in Proc. Int. Symp. Wireless Personal Multimedia Communications $(W P M C)$, Lapland, Finland, 2008.

[3] D. Ferling, T. Bohn, D. Zeller, P. Frenger, I. Godor, Y. Jading, and W. Tomaselli, "Energy Efficiency Approaches for Radio Nodes (submitted)," in Future Network \& Mobile Summit, Florence, Italy, 2010.

[4] "EU Commissioner calls on ICT industry to reduce its carbon footprint by $20 \%$ as early as 2015," 2009. [Online]. Available: http: //europa.eu/rapid/pressReleasesAction.do?reference=MEMO/09/140

[5] Ericsson, "Long Term Evolution (LTE): an introduction," 2007. [Online]. Available: http://www.ericsson.com/technology/whitepapers/ lte_overview.pdf

[6] B. Gorini, "ETSI work programme on energy saving, presented at Telecommunications Energy Conference," 2007.

[7] V. Rodoplu and T. Meng, "Bits-per-Joule Capacity of Energy-Limited Wireless Networks," IEEE Transactions on Wireless Communications, vol. 6, no. 3, pp. 857-865, 2007.

[8] J. L. Gao, "Analysis of Energy Consumption for Ad Hoc Wireless Sensor Networks Using a Bit-Meter-per-Joule Metric," 2002.

[9] G. Miao, N. Himayat, and Y. Li, "Energy-efficient transmission in frequency-selective channels," in Proc. IEEE Globecom, 2008.

[10] Ericsson, "Sustainable energy use in mobile communications," 2007. [Online]. Available: http://www.ericsson.com/technology/whitepapers/ sustainable_energy.pdf

[11] S. Cui, A. Goldsmith, and A. Bahai, "Energy-Efficiency of MIMO and Cooperative MIMO Techniques in Sensor Networks," IEEE Journal on Selected Areas in Communications, vol. 22, no. 6, pp. 1089-1098, 2004.

[12] T. Himsoon, W. Siriwongpairat, and K. Liu, Energy-efficient cooperative transmission over multiuser OFDM networks: who helps whom and how to cooperate2. IEEE, 2005.

[13] L. Hérault, "Sustainability of mobile network solutions," Santander, Spain, 2009.

[14] L. Hérault, E. Calvanese Strinati, D. Zeller, O. Blume, M. A. Imran, R. Tafazolli, J. Lundsjö, Y. Jading, and M. Meyer, "Green Communications: A Global Environmental Challenge," in Proc. Int. Symp. Wireless Personal Multimedia Communications (WPMC), Sendai, Japan, 2009.

[15] H. Claussen, L. T. W. Ho, and F. Pivit, "Leveraging advances in mobile broadband technology to improve environmental sustainability," Telecommunications Journal of Australia, vol. vol59, 2009.

[16] — Effects of joint macrocell and residential picocell deployment on the network energy efficiency. IEEE, 2008.

[17] E. Bogenfeld and I. Gaspard, "A White Paper by the FP7 project End-to-End Efficiency ( E 3 ) Self-x in Radio Access Networks," Framework, pp. 1-24, 2008. [Online]. Available: https://www.ict-e3.eu/ project/white_papers/Self-x_WhitePaper_Final_v1.0.pdf

[18] H. Karl, "An overview of energy efficiency techniques for mobile communication systems," Berlin, 2003.

[19] P. Herhold, W. Rave, and G. Fettweis, "Relaying in CDMA networks: Pathloss reduction and transmit power savings," , in Proc. IEEE Vehicular Technology Conference (VTC), 2003.

[20] A. Radwan and H. S. Hassanein, NXG04-3: Does Multi-hop Communication Extend the Battery Life of Mobile Terminals? IEEE, 2006.

[21] EU FP6 IP, "Ambient Networks," 2007. [Online]. Available: http: //www.ambient-networks.org 